\title{
A Randomised control study on effects of autologous platelet rich plasma injection in early osteoarthritis knee by single dose versus double dose regimen
}

\author{
K. Deepak ${ }^{1}$, Hameed Reza, ${ }^{2, *}$ G. Jagadesh ${ }^{3}$, Akash ${ }^{4}$, Pavan Kumar ${ }^{5}$ \\ ${ }^{1,3,4}$ M.S, ${ }^{2,5} \mathrm{DNB}$, Dept. of Orthopaedics, Balaji Institute of Surgery, Research and Rehabilitation for the Disabled (BIRRD), \\ Andhra Pradesh, India
}

*Corresponding Author:

Email: hamureza786@gmail.com

\begin{abstract}
Materials and Methods: A total of 263 knees, (150 patients), males 65, females 85 with early OA were divided randomly into two groups. Group A 75 patients (134 knees) received a single injection of PRP, group B 75 patients (129 knees) received 2 injections of PRP 3 weeks ap art. PRP with a platelet count 5 times that of baseline was administered in all. Clinical outcome was evaluated using the Western Ontario and McMaster Universities Arthritis Index (WOM AC) questionnaire before treatment and at 1 month, 3 month, and 6 months post injection. A reduction in WOM AC score is suggestive of improvement in patient condition. Results: significant improvement in all WOM AC parameters was noted in groups A and B within 3 to 4 weeks and lasting until the final follow-up at 6 months. The mean WOM AC scores (pain, stiffness, physical function, and total score) for group A at baseline were 15.8, 6.22, 43.09, and 65.11, respectively, and at final follow-up were 4.83, 1.00, 14.12, and 19.95, respectively, showing significant improvement. Similar improvement was noted in group B (mean WOM AC scores at baseline: 16.51, 6.91, 41.98, and 65.38, respectively; mean WOM AC scores at final follow up: 5.50,1.12, 13.69, and 20.37, respectively. The 2 groups were compared with each other, and no improvement was noted in group B as compared with groups A (P, value 0.77 ).

Conclusion: Autologous PRP infiltration in early Osteoarthritis of Ahlback's radiological grading does give relief from pain, stiffness and improves functionality without any major side effects. Double dose doesn't offer any additional advantage.
\end{abstract}

Keywords: Platelet rich plasma, Early osteoarthritis knee.

\section{Introduction}

Osteoarthritis of knee is the major cause of disability. Osteoarthritis is a chronic disorder of synovial joints in which there is progressive softening and disintegration of articular cartilage. Osteoarthritis is a dynamic phenomenon, it shows features of both destruction and repair. Osteoarthritis is the fourth leading cause of 'years lived with disability' (YLD), accounting for $3.0 \%$ of total global YLD's. As per WHO by 2030, the demand for total knee arthroplasties will increase up $67 \%$.

Osteoarthritis is clinically heterogeneous, and the processes that cause deterioration are still poorly understood. ${ }^{1}$ Current opinion is that the disease progression results from an imbalance between proinflammatory cytokines (including interleukin [IL]1a, IL-1, and tumor necrosis factor-1 and antiinflammatory cytokines (including IL-4, IL-10, and IL1ra).This cytokine imbalance is thought to activate proteolytic enzymes, leading to the destruction of cartilage. Autologous platelet rich plasma contains a bunch of growth factors, it act as a medium providing multiple growth factors needed for management of Osteoarthritis. PRP has increased concentrations of PDGF, VEGF, TGF-beta1, and EGF compared with their concentrations in whole blood. PRP in OA knee joints delivers natural growth factors and their cytokines containing anabolic and catabolic factors in supraphysiologic concentrations directly into the site of injury to potentially optimize the healing environment. Maintaining a natural ratio of growth factors may provide a homeostatic environment and theoretically provide an abundance of healing factors without disrupting their in vivo relationships. ${ }^{43}$

\section{Materials and Methods}

It is a prospective randomized study conducted on 263 primary osteoarthritic knee joints diagnosed and staged as per Ahlback radiological grading, selected from the Outpatient Department of Orthopaedics, Balaji Institute of Surgery, Research and Rehabilitation for the Disabled (BIRRD). Clinical examination and $x$ rays of the knee joints were done and blood sample of the patients were collected and PRP prepared in the Department of Transfusion Medicine of the same institute. By computer based randomization, patients are divided into two groups group A and group B. Group B received two doses of PRP 3 weeks apart, group A received single dose of prp. Infiltration was done in Operation Theatre under strict aseptic conditions. Patients were assessed with WOMAC (Western Ontario McMaster Universities Arthritis Index) scoring pre injection of PRP and post injection period of 1 month, 3 month and 6 months. A reduction in WOMAC score is suggestive of improvement in the patient's condition.

PRP Preparation and Interventional Procedure: In the Department of Transfusion Medicine, from each patient $50 \mathrm{ml}$ of venous blood was collected from the antecubital vein atraumatically in an effort to avoid irritation and trauma to the platelets with a syringe, blood was transferred to the vacutainers of $4.5 \mathrm{ml}$ 
containing CPD-A1 (citrate phosphate dextrose and adenine) as an anticoagulant. The tubes were then centrifuged for 15 minutes at $1500 \mathrm{rpm}$ on a table-top centrifuge, and the blood was separated into PRP and residual red blood cells. The PRP was then extracted through a pipette and transferred to a test tube. The final PRP was assessed for platelet count and was supplied for injection in a $10-\mathrm{mL}$ syringe (approximately $5 \mathrm{~mL}$ per knee). Total leucocyte count and platelet count were measured from the patient's peripheral blood as well as in the final PRP. Total leucocyte count was zero in our PRP, The mean platelet count achieved by our method was more than five times the platelet count of blood of that patient.

In the operation theatre, with the patient in supine position, knee was scrubbed, painted and draped with sterile towels. With the patients knee in 45-90 degrees of flexion so that joint is opened for injection through lateral parapatellar (anterolateral portal) approach. Under aseptic conditions, $5 \mathrm{~mL}$ platelet concentrate was injected into the knee joint with an 18-gauge needle without local anesthetic. After the procedure, Jone's compression bandage was applied and the knees were immobilized for 10 minutes. Patients were observed for 30 minutes for any possible side effects like dizziness, sweating. During the follow-up period, nonsteroidal anti-inflammatory drugs were not allowed, and tramadol (dosage, $50 \mathrm{mg}$ bds) was prescribed in case of discomfort; all patients were asked to stop medications 48 hours before follow-up as sessment.

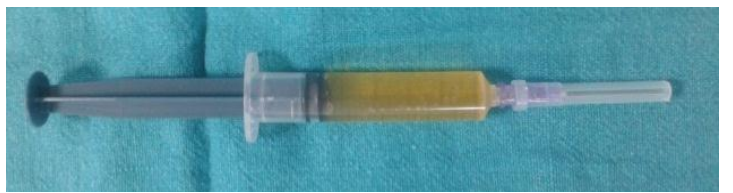

Fig. 1: 5ml of PRP in syringe

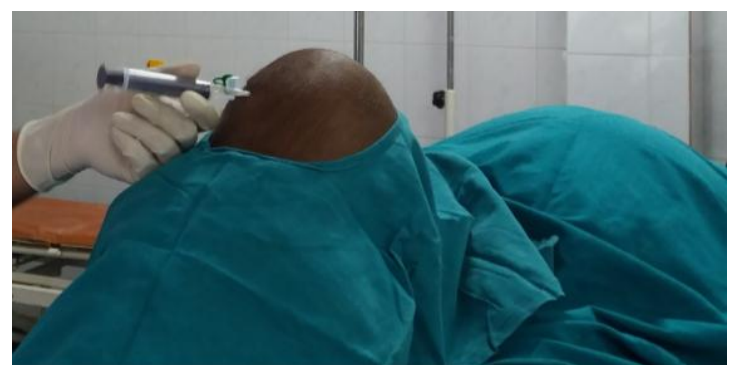

Fig. 2: Infiltartion of PRP into Rt knee

Statistical Analysis: The collected data been computed in excel and analysed through SPSS version 2.0, EPI. INFO, $\mathrm{T}$ test been used and $\mathrm{p}$ value and mean values been obtained. The descriptive statistics (eg, mean, standard deviation) for normally distributed parameters were calculated for two groups. The normally distributed parameters were compared for their means. A $P$ value of $<.05$ was considered to be significant in all tests.

\section{Results \\ Pain Parameter: In groups A and B, the mean pain score decreased frombas eline at 4-week and 3-month follow-up, which was significant followed by a slight increase in pain at the 6-month follow-up, which was not significant. However, the mean pain at 6 months was still less than that at baseline. The improvement was maintained from the end of the therapy to 6 months' follow-up, with only slight worsening at 6 months. As there is no difference in $\mathrm{P}$ value of group A and group B there is no difference between group A and group B. \\ Similar pattern of results were found in secondary parameters of WOMAC (stiffnes s, physical function, total WOMAC score) that of pain.}

Table 1:

\begin{tabular}{|l|c|c|}
\hline \multicolumn{1}{|c|}{ WOMAC Parameter } & $\begin{array}{c}\text { Group A } \\
\text { 0 1mnth 3mnth 6mnth }\end{array}$ & $\begin{array}{c}\text { Group B } \\
\text { 0 1mth 3mth 6mth }\end{array}$ \\
\hline $\begin{array}{l}\text { Mean } \\
\begin{array}{l}\text { Pain } \\
\text { P value }\end{array}\end{array}$ & 14.811 .718 .34 .8 & 15.8912 .959 .65 \\
\hline $\begin{array}{l}\text { Mean } \\
\begin{array}{l}\text { Stiffness } \\
\text { p value }\end{array}\end{array}$ & 0.0130 .0010 .0030 .632 & 0.0120 .000 .0030 .632 \\
\hline $\begin{array}{l}\text { Mean } \\
\begin{array}{l}\text { Functionality } \\
\text { p value }\end{array}\end{array} \quad 6.224 .182 .41 .00$ & 6.914 .882 .861 .12 \\
\hline $\begin{array}{l}\text { Mean } \\
\text { Total WOMAC } \\
\text { p value }\end{array}$ & 0.0030 .0070 .030 .444 & 0.0030 .0070 .030 .444 \\
\hline
\end{tabular}

\section{Discussion}

Articular cartilage lesions and degeneration are difficult to treat and present a challenge for orthopaedic surgeons because of the distinctive structure and function of hyaline cartilage and its inherent low healing potential. For therapeutic intervention, laboratory investigations are focusing on the possibility of preserving normal homeostasis or blocking or at least 
delay the need for more invasive surgical procedures. Current pharmacologic interventions may only temporarily reduce chronic pain, but for the time being, no proven disease modifying therapy is available. ${ }^{22}$

In this prospective randomised study, patients were randomized into two groups, one for single dose regimen, other for double dose regimen WOMAC scores were evaluated pre-injection and post-injection period on first month, three months and sixth months. There is a correlation in Grade I and Grade II mean WOMAC scores. In Grade I, the mean WOMAC score of pain, stiffness and functionality is lower than the Grade II osteoarthritis knee joints. There was no control group in this study. The number of platelets used are more than 5 times the base line, as all the patients selected were having more than one lakh platelets, so every patient got more than 5 lakh platelets per $\mathrm{ml}$, which is prepared by single spinning of the sample for 15 minutes with 1500 RPM (Rotations per minute) and leucofilters were not used. Patel et al in 2013, used single spinning technique with leuco-filters. They have given two injections of PRP activated with $\mathrm{CaCl}_{2}$, each $8 \mathrm{ml}$, with 3 weeks gap. Their platelet count is less than 5 times the base line. ${ }^{1,22}$ In 2011, Filardo et al, used 5 $\mathrm{ml} \mathrm{PRP}$ with 5 times the platelet count prepared from double spinning technique and activated with $\mathrm{CaCl}_{2}$. They have infiltrated three injections of PRP with one week gap. ${ }^{11}$ The preparation of PRP, number of platelets, amount of PRP infiltrated, and frequency of injections were not uniform.

In this study, all the patients have shown decrease in the WOMAC score. Their mean pain, stiffness and functionality scores have decreased. The decrease in WOMAC score continued upto six months. The improvement in our patients could be explained by the fact that injected platelets might have acted at different levels and were stimulating the chondral anabolism or slowing the catabolic process.

As we have given a working classification to assess the results, in group A, 15 joints have shown excellent results, 76 joints have shown good results, 27 joints have shown fair results and 16 joints have shown poor results. In group B, 13 joints have shown excellent results, 69 joints have shown good results, 31 joints have shown fair results and 16 joints have shown poor results though the mean pain scores have decreased in all the patients, the efficacy has been varied from patient to patient. Results were poor in obese, patients with active manual labour. The results have shown better improvement in grade I osteoarthritis knee joints than grade II knee joints. Grade I patients shown 57.5 percentage of improvement, whereas grade II patients shown 52.9 percentage when evaluated with WOMAC score. But the difference is not statistically significant. In every patient, there is decrease in WOMAC score, but in no one it has reached ' 0 '. It means that PRP delays the osteoarthritic progression in the joints, but it has not cured osteoarthritis. To evaluate its duration of action long term follow up studies are required. In this study the results have shown that the effect of PRP sustained for 6 months with continuous decrease in all parameters, i.e. pain, stiffness and functionality of the WOMAC score. Filardo et al. in 2012 found that there was worsening of the condition of the patients from the end of 9 months, implying that the duration of action of PRP was 9 months. But, the longevity of the benefits of PRP cannot be emphatically established by our study as the follow up is only short term, long term studies are necessary to study this aspect.

Immediate post infiltration, all patients have complained of severe pain but no systemic and long term complications noted during the course of our study. Sandeep Patel et al, n 2013, in their study have documented some systemic adverse effects. Which were immediate and systemic rather than local and were of short duration not lasting more than 30 minutes. But they have not explained the characteristics of the adverse effects. They have attributed these adverse effects to the higher number of platelets in the infiltrating PRP sample and the possibility of $\mathrm{CaCl}_{2}$, which was used as an activating agent. ${ }^{1}$ Kon et al. in 2010 and Sanchez et al. in 2007 have reported some injection pain, local inflammation of short duration and reaccumulation of effusion, but the exact numbers were not mentioned. ${ }^{25,48}$

Study Limitations: The age, Body Mass Index (BMI), were not considered in selecting the patients. Cartilage mapping was not done because of its cost. No predefined classification system was there, though we have given a working classification to assess the results. Study follow up period was maximum for six months, few were followed further up, it would have given more understanding on longevity if it was followed for longer periods. Further studies are required to better understand the mechanism of action of PRP, the dosage of PRP, duration of action, frequency of injections, its composition and role of $\mathrm{CaCl}_{2}$ in its activation. It is necessary to understand the results of PRP, whether they are temporary or permanent. Different platelet concentrations and application modalities have to be studied further.

\section{Conclusion}

We can safely conclude that Autologous PRP infiltration in early Osteoarthritis (Grade I and Grade II) of Ahlback's radiological grading does give relief from pain, stiffness and improves functionality without any major side effects and can be recommended as a viable modality of treatment. The efficacy wears off early in those who continue to pursue heavy manual labour on comparision to those with sedentary lifestyle. But there is no significant difference between single dose and double dose regimen, we conclude that double dose doesn't offer any additional advantage.

Funding: No funding sources 
Conflict of Interest: None declared

Ethical Approval: The study was approved by institutional ethics committee.

\section{References}

1. Sandeep Patel, Mandeep S. Dhillon, Sameer Aggarwal, Neelam Marwaha, and Ashish Jain. Treatment with Platelet-Rich Plasma Is More Effective than Placebo for Knee Osteoarthritis - A Prospective, Double-Blind, Randomized Trial. The Am J Sports Med 2013;41:356-64.

2. Anna Litwic, Mark H Edwards, Elaine M Dennison, and Cyrus Cooper. Epidemiology and burden of Osteoarthritis, British medical bulletin 2015:1-15.

3. Steven Sampson, Marty Reed, Holly Silvers, Michael Meng, Bert Mandelbaum. Injection of Platelet-Rich Plasma in Patients with Primary and Secondary Knee Osteoarthritis- A Pilot Study, American Journal of Physical Medicine \& Rehabilitation, 2010:1961-69.

4. Pietrzak WS, Eppley BL: Platelet rich plasma: Biology and new technology. J Craniofac Surg 2005;16:1043-54.

5. Eppley BL, Woodell JE, Higgins J: Platelet quantification and growth factor analysis from platelet-rich plasma: Implications for wound healing. Plast Reconstr Surg 2004;114:1502-7.

6. Werner S, Grose R: Regulation of wound healing by growth factors and cytokines. Physiol Rev 2003;83:83570.

7. Anitua M, Sanchez E, Nurden A, et al: New insights into and novel applications for platelet -rich fibrin therapies. Trends Biotechnol 2006;24:227-34.

8. Sampson S, Gerhardt M, Mandelaum B: Platelet rich plasma injection grafts for musculoskeletal injuries: A review. Curr Rev Musculoskelet Med 2008;1:165-74.

9. Mishra A, Pavelko T: Treatment of chronic elbow tendinosis with buffered platelet rich plasma. Am J Sports Med 2006;10:1-5 15 .

10. Barrett S, Erredge S: Growth factors for chronic plantar fasciitis. Podiatry Today 2004;17:37-42.

11. Giuseppe Filardo, ElizavetaKon, Roberto Buda, Antonio Timoncini, Alessandro Di Martino, Annarita Cenacchi, Pier Maria Fornasari, SandroGiannini, MaurilioMarcacci. Platelet-rich plasma intra-articular knee injections for the treatment of degenerative cartilage lesions and osteoarthritis. Knee Surg Sports Traumatol Arthrosc (2011) 19:528-535.

12. J. Alsousou, M. Thompson, P. Hulley, A. Noble, K. Willett (2009) the biology of platelet-rich plasma and its application in trauma and orthopaedic surgery-A review of the literature. The journal of bone and joint surgery 2009;91:987-96.

13. Gibble J, Ness P. Fibrin glue: the perfect operative sealant? Transfusion 1990;30:741-47.

14. Robert E. Marx, Eric R. Carlson, Ralph M. Eichstaedt, Steven R. Schimmele, James E. Strauss, Karen R. Georgeff. Platelet rich plasma-Growth factor enhancement for bone grafts. Oral Med Oral Pathol Oral Radiol Endod 1998;85:638-46.

15. Hammond JW, Hinton RY, Curl LA, Muriel JM, Lovering RM. Use of autologous platelet-rich plasma to treat muscle strain injuries. Am J Sports Med. 2009;37(6):1135-1142.

16. Kajikawa Y, Morihara T, Sakamoto H. Platelet-rich plasma enhances the initial mobilization of circulationderived cells for tendon healing. J Cell Physiol 2008;1-3.
17. Lucarelli E, Fini M, Beccheroni A. Stromal stem cells and platelet-rich plasma improve bone allograft integration. Clin Orthop 2005;435:62-8.

18. Tomoyasu A, Higashio K, Kanomata K. Platelet-rich plasma stimulates osteoblastic differentiation in the presence of BMPs. Biochem Biophys Res Commun 2007;361:62-7.

19. Peitro S. Randelli, Paolo Arrigoni, Paolo Cabitza, PieroVolpi, Nicola Maffulli. Autologous platelet rich plasma for arthroscopic rotator cuff repair: a pilot study. Disability and Rehabililitation 2008; 30:1584-589.

20. MontoRR.Platelet rich plasma treatment for Chronic Achilles tendinosis. Foot Ankle Int 2012;33:379-85.

21. Spakova T, Rosocha J, Lacko M, Harvanova D, Gharaibeh A. Treatment of knee joint osteoarthritis with autologous platelet-rich plasma in comparison with hyaluronic acid. Am J Phys Med Rehabil 2012;91:411417.

22. Elizaveta Kon, Bert Mandelbaum, Roberto Buda, Giuseppe Filardo, Marco Delcogliano, Antonio Timoncini, Pier Maria Fornasari, Sandro Giannini, Maurilio Marcacci. Platelet-Rich Plasma Intra-Articular Injection versus Hyaluronic Acid Viscosupplementation as Treatments for Cartilage Pathology: From Early Degeneration to Osteoarthritis. Arthroscopy 2011;11:1490-1501.

23. Cerza, Carni S, Carcangiu A, Di Vavo I, Schiavilla V, Pecora A, De Biasi G, Ciuffreda M. Comparison between hyaluronic acid and platelet-rich plasma, intraarticular infiltration in the treatment of gonarthrosis. Am J Sports Med. 2012 Dec;40(12):2822-7.

24. Ana Wang-Saegusa, RamónCugat, Oscar Ares, Roberto Seijas, Xavier Cuso. Montserrat Garcia-Balletbo. Infiltration of plasma rich in growth factors for osteoarthrit is of the knee short-term effects on function and quality of life. Arch Orthop Trauma Surg 2010;36:1345-1351.

25. Mikel Sánchez, EduaroAnitua, Juan Azofra, Isabel Andia, Sabino Padilla, InigoMujika. Comparison of surgically repaired Achilles tendon tears using platelet-rich fibrin matrices. Am J Sports Med2007; 35:245-51.

26. Giuseppe Filardo, ElizavetaKon, Stefano Della Villa, Ferruccio Vincentelli, Pier Maria Fornasari, Maurilio Marcacci. Use of platelet-rich plasma for the treatment of refractory jumper's knee. International Orthopaedics 2010;34:909-15.

27. Gomez et al. Ultrasound-guided platelet-rich plasma injections for the treat ment of osteoarthritis of the hip. Rheumatology (Oxford).2012;51:144-50.

28. Randelli P, Arrigoni P, Ragone V, Aliprandi A, Cabitza P. Platelet rich plasma in arthroscopic rotat or cuff repair: A prospective RCT study, 2-year follow up. J Shoulder Elbow Surg. 2011;20:518-28.

29. Rha DW, Park GY, Kim YK, Kim MT, Lee SC. Comparison of the therapeutic effects of ultrasound-guided platelet-rich plasma injection and dry needling in rotator cuff disease: A randomized controlled trial. ClinRehabil. 2013;27:113-22.

30. Wery GL, Kulkarni S, Pennisi AE. Use of autologous growth factors in lumbar spinal fusion. Bone 1999;25:4750 .

31. Joost C. Peerbooms, Jordi Sluimer, Daniel J. Bruijn, Taco Gosens. Positive effect of an autologous platelet concentrate in lateral epicondylitis in a double-blind randomized controlled trial. The American Journal of Sports Medicine 2010;38:255-62.

32. Owens RF, Jr, Ginnetti J, Conti SF, Latona C. Clinical and magnetic resonance imaging outcomes following 
platelet rich plasma injection for chronic mid-substance Achilles tendinopathy. Foot Ankle Int. 2011;32:1032-9

33. deJonge S, de Vos RJ, Weir A, van Schie HT, BiermaZeinstra SM, Verhaar JA, et al. One-year followup of platelet-rich plasma treatment in chronic Achilles tendinopathy: A double-blind randomized placebocontrolled trial. Am J Sports Med. 2011;39:1623-9.

34. Monto RR. Platelet rich plasma treatment for chronic Achilles tendinosis. Foot Ankle Int.2012;33:379-85.

35. Schepull T, Kvist J, Norrman H, Trinks M, Berlin G, Aspenberg P. Autologous platelets have no effect on the healing of human achilles tendon ruptures: A randomized single-blind study. Am J Sports Med.2011;39:38-47.

36. Filardo G, Kon E, Della Villa S, Vincentelli F, Fornasari PM, Marcacci M. Use of platelet-rich plasma for the treatment of refractory jumper's knee. Int Orthop. 2010;34:909-15.

37. Gosens T, Den Oudsten BL, Fievez E, van't Spijker P, Fievez A. Pain and activity levels before and after platelet-rich plasma injection treatment of patellar tendinopathy: A prospective cohort study and the influence of previous treatments. Int Orthop. 2012;36:1941-6.

38. Karsdal MA, et al. should subchondral bone turnover be targeted when treating osteoarthritis? Osteoarthritis Cartilage. 2008; 16(6):638-46.

39. P. Lavenge et al. Subchondral and trabecular bone metabolism regulation in canine experimental knee osteoarthritis. Osteo Arthritis and Cartilage (2005) 13, 310-317.

40. Boyd SK, Muller R, Zernicke RF. Mechanical and architectural bone adaptation in early stage experimental ost eoarthritis. J Bone Miner Res. 2002; 17(4):687-94.

41. Martel-Pelletier J. Pathophy siology of osteoarthritis. Osteoarthritis Cartilage. 1999;7(4):371-3.

42. A Mahajan, S Verma, V Tandon. Osteoarthritis. JAPI.2005; 53:634-641

43. Brian J. Cole, Shane T. Seroyer, Giuseppe Filardo, Sarvottam Bajaj and Lisa A. Fortier. Platelet-Rich Plasma: Where are we now and where are we going? Sports health 2010;3:203-210.

44. The Journal of Rheumatology [Internet]. Nicholas Bellamy: WOMAC: A 20-Year Experiential Review of a Patient-Centered Self-Reported Health Status Questionnaire; c 2002. Editorial; Dec,2002. Available from: https://jrheum.com/subscribers/02/12/2473.html American College of Rheumatology [Internet]. Atlanta. American College of Rheumatology;2015. [cited 2015]. Available from: http://www.rheumatology.org/I-AmA/Rheumatologist/Research/ClinicianResearchers/Western-Ontario-McMaster-UniversitiesOsteoarthritis-Index-WOMAC

45. Vaquerizo V, Placencia MA, Arribas I, et al. Comparison Comparison of intra-articular injections of plasma rich in growth factors versus Durolane Durolane hyaluronic acid in thetreatment of patients with symptomatic osteoarthritis: a randomized controlled trail. Arthroscopy 2013;29:1635-43.

46. Filardo G, Kon E, Di Martino A, et al. Platelet-rich plasma versus hyaluronic acid to treat knee degenerative pathology: study design and preliminary results of arandomized controlled trail. BMC Musculoskelet Disord 2012;213:229.

47. Kon E, Buda R, Filardo G, et al. Platelet-rich Plasma: intra-articular knee injections produced favorable results on degenerative cartilage lesions. Knee surg Sports traumatol Arthrosc 2010;18:472-479. 